\title{
Short-term effects of smoking marijuana on balance in patients with multiple sclerosis and normal volunteers
}

\begin{abstract}
A double-blind randomized placebo-controlled study of inhaled marijuana smoke on postural responses was performed in 10 adult patients with spastic multiple sclerosis (MS) and 10 normal volunteers matched as closely as possible for age, sex, and weight. A computer-controlled dynamic posturographic platform with a video line scan camera measured shoulder displacement in response to pseudorandom platform movements. Premarijuana smoking patient tracking was inferior to that of the normal volunteers as indicated by the higher noise variance of the former. Smoking one marijuana cigarette containing $1.54 \% \Delta^{9}$-tetrahydrocannabinol increased postural tracking error in both the patients and normal control subjects with both eyes open and closed; this untoward effect was greatest for the patients. The tracking error was also accompanied by a decrease in response speed for the patients with their eyes closed. Marijuana smoking further impairs posture and balance in patients with spastic MS. (CLIN PHARMACOL THER 1994;55:324-8.)
\end{abstract}

\section{Harry S. Greenberg, MD, Susan A. S. Werness, PhD, James E. Pugh, MD, Robert O. Andrus, PhD, David J. Anderson, PhD, and Edward F. Domino, MD Ann Arbor, Mich.}

Anecdotal reports frequently suggest that smoking marijuana has beneficial effects in spasticity. Consroe and Snider ${ }^{1}$ and Consroe and Sandyk ${ }^{2}$ reviewed the scientific literature regarding the effects of marijuana and cannabinoids in various neurologic states, including spasticity; mostly beneficial results were reported (see their references 144 to 146 and 148 to 150 ). These clinical studies contrast with the dose-response study by Kipliner et al. ${ }^{3}$ in which marijuana smoking in a linear dose-response manner produced a deterioration of mental, motor, and postural stance on a wobble board in normal volunteers.

Postural regulation is a complex motor function that is dependent on the integration of several sensory modalities and neuronal mechanisms at spinal, cerebral,

From the Departments of Neurology, Otolaryngology, and Pharmacology, University of Michigan Medical Center.

Supported in part by a grant from the National Multiple Sclerosis Society (New York, N.Y.) and grant 361024 from the Neuropsychopharmacology Research Fund, University of Michigan (Ann Arbor, Mich.)

Received for publication April 29, 1993; accepted Sept. 23, 1993. Reprint requests: Harry S. Greenberg, MD, Department of Neurology, University of Michigan, Ann Arbor, MI 48109-0316.

Copyright (C) 1994 by Mosby-Year Book, Inc.

$0009-9236 / 94 / \$ 3.00+0 \quad 13 / 1 / 51654$ and cerebellar levels. Failure to maintain postural control is an indication of a dysfunction in one or more of the subsystems involved. A decrease in postural control is a sensitive index of undesirable motor effects of potential therapeutic antispastic agents, including use of marijuana by humans.

The hypothesis of this study was that marijuana smoking would relieve the spasticity of patients with multiple sclerosis (MS) sufficiently to allow better postural control and thus produce a therapeutic benefit. Therefore neurologically normal subjects and patients with MS matched for age, weight, and height were studied with use of a microcomputer-driven platform to measure postural stance. Pharmacokinetic or pharmacodynamic measures were not obtained because the possible therapeutic benefit or side effects of single exposure to marijuana smoke was of special interest.

\section{METHODS}

Testing apparatus. The posture measurement system consisted of a stimulus delivery component, a response measurement component, and a microcomputer with an appropriate amount of memory and necessary interfaces for data acquisition and analysis. The balance perturbation was delivered by way of a 


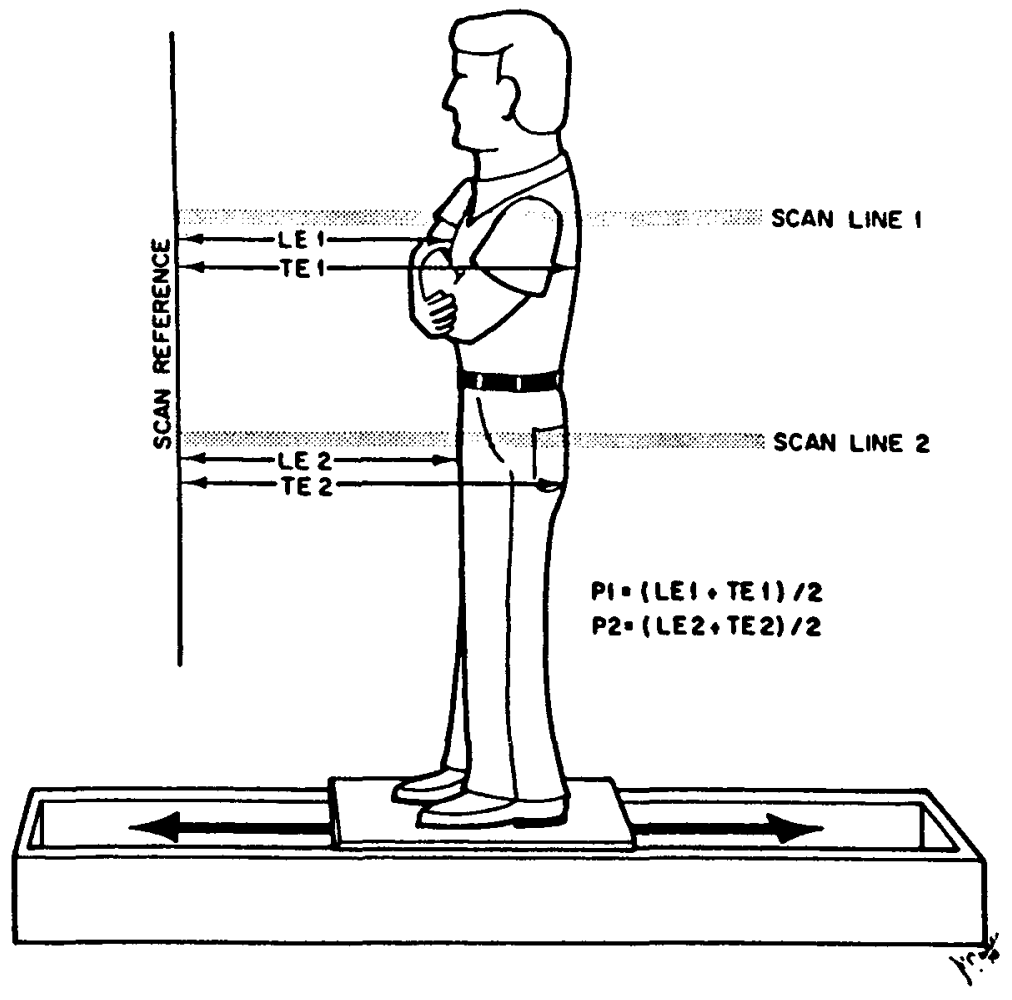

Fig. 1. Schematic diagram of platform with video line scan cameras focused on shoulders and hips of patient.

translating platform. ${ }^{4}$ Fig. 1 illustrates the platform with a subject on board. The response measurement portion of the system was composed of two digital line scan cameras that sensed the average position of the sagittal plane body silhouette of the subject standing on the moving platform in a configuration for induced anteroposterior sway. One camera scanned horizontally at the shoulder level while the other scanned at the waist. The line scan camera measurements of waist and shoulder position were resolvable to the nearest $0.175 \mathrm{~cm}$. Mass storage of data was provided by floppy discs. The characteristics of the posture measurement system and its application have been described in detail elsewhere ${ }^{4-8}$; therefore only a brief description is provided here.

Stimulus characteristics. Pseudorandom displacement stimuli were generated for each trial. Stimuli of $7.5 \mathrm{~cm} / \mathrm{sec}$ peak velocity of motion were used.

Measured variables. Twenty seconds of actively filtered measurements for the shoulder, waist, and platform positions were sampled and stored on floppy discs at a rate of $25 \mathrm{~Hz}$ per channel. In the models derived, the measured platform position was the deterministic input time series, whereas the measured shoulder and waist positions comprised the output time series. In both the eyes-open and eyes-closed trials, the subjects' postural responses usually showed a transient period of a few seconds in which the response characteristics were significantly changing, followed by a less variable steady-state phase. Only the steady-state phase, the last 10 seconds (eyes closed) or the last 14 seconds (eyes open), was used in the data analysis before and immediately after marijuana smoking.

Experimental protocol. This study was approved by the University of Michigan Medical Center Institutional Review Board for Research Involving Human Subjects. Before the first experimental session and after explanation of the testing procedures, an informed consent was signed by each volunteer. Each subject was tested in a double-blind trial on 3 consecutive days. On 2 of those days, the subject received active marijuana on one day and placebo on the other. A single marijuana $\left(1.54 \% \Delta^{9}\right.$-tetrahydrocannabinol $\left[\Delta^{9}\right.$ THC]) or alcohol-extracted marijuana placebo cigarette was smoked and inhaled by each subject taking deep breaths over 10 minutes. Ten minutes after completion of smoking, each subject control was tested on 
Table I. Significant normal subject and patient posture model parameters in ANOVA analysis*

\begin{tabular}{|c|c|c|c|c|c|c|}
\hline \multirow[b]{2}{*}{ Factor } & \multicolumn{2}{|c|}{ Patients } & \multicolumn{2}{|c|}{ Normal subjects } & \multicolumn{2}{|c|}{ Significance level } \\
\hline & Eyes open & Eyes closed & Eyes open & Eyes closed & Healtht & Eyes $\neq$ \\
\hline Dead time & 8.12 & 7.96 & 6.05 & 6.29 & 0.0001 & NS \\
\hline Signal variance & $0.389 \S$ & $5.06 \S$ & $0.071 \S$ & $3.43 \S$ & 0.0054 & 0.0000 \\
\hline Noise variance & $0.979 \S$ & $3.78 \S$ & $0.580 \S$ & $2.04 \S$ & 0.007 & 0.0000 \\
\hline $1.4 \mathrm{~Hz}$ phase lag & 4.11 & 4.15 & 4.33 & 3.53 & 0.0359 & 0.0274 \\
\hline $0.2 \mathrm{~Hz}$ gain & 1.32 & 2.30 & 0.581 & 2.09 & NS & 0.0000 \\
\hline SNR & $0.475 \S$ & $1.14 \S$ & $0.147 \S$ & $1.59 \S$ & NS & 0.0000 \\
\hline Residual & 0.020 & 0.034 & 0.020 & 0.028 & NS & 0.0001 \\
\hline
\end{tabular}

Not significant; $0.2 \mathrm{~Hz}$ gain, low frequency transfer function gain; SNR, signal to noise ratio.

* Numbers in this table are group means, except where indicated.

${ }^{+}$Health, Patients versus normal subjects for factor in ANOVA analysis

Eyes, Eyes-open versus eyes-closed for factor in ANOVA analysis.

§Median.

the platform at about the time a peak plasma concentration of $\Delta^{9}$-THC is expected after inhalation. ${ }^{9}$ Rest periods were provided at the beginning of a session and every 20 minutes throughout. The subject was instructed to stand in his or her stocking feet, at a 45 degree angle, heels together, head upright, arms comfortably folded across the chest, and knees locked. Ear protectors were worn to minimize the effects of any background noise. The subject's eyes were either open and fixed on a target at eye level or closed, depending on the requirements of the specific trials. Each session consisted of twenty 20 -second trials. These trials, randomly mixed by a computer program, were either static (no platform motion), transient, or pseudorandom in nature. Shoulder responses (before treatment and after treatment) in the eyes-open and eyes-closed paradigm from the placebo and the second of the 2 drug days were analyzed, yielding a total of 160 postural response measures. Only pseudorandom movement in patients and control subjects in the eyes-open and eyes-closed paradigms were analyzed focusing on the shoulder response.

Patients. All 10 patients had MS by clinical and laboratory criteria with the predominant clinical deficit a spastic myelopathy (Table I). There were five male and five female patients with a median age of 32 years (range, 21 to 55 years). All patients had a stiff-legged gait with circumduction of at least one leg. Four patients were subjectively classified as having mild and six moderate gait dysfunction. Eight patients had increased lower extremity tone or reflexes or both. Seven patients had bilaterally present Babinski responses and one patient had unilaterally present Babinski responses. Six patients had a decrease in lower extremity sensory modalities and two had a sensory pin level in the thoracic area. All 10 patients had mild definite secondary areas of involvement of their nervous system other than the spinal cord, two with optic nerve involvement, two with brain stem dysfunction, and nine with cerebellar or brain stem dysfunction. Ten normal subjects without cardiac, rheumatologic, or neurologic disease were matched for sex, age, weight, and height to the 10 patients with multiple sclerosis with no age variance of greater than 3 years, weight variance greater than 10 pounds, or height variance greater than 2 inches.

Mathematical analysis. A linear time invariant difference equation model was postulated to be appropriate for the steady-state portion of the pseudorandom responses. The mathematic model and computer program have been described in detail elsewhere. ${ }^{7,8}$

Statistical analyses. The postural response model data were subjected to an ANOVA analysis to determine statistically significant effects attributable to the treatment, MS/normal subject factor, the presence or absence of vision, or the sex of the subject.

\section{RESULTS}

The patients and control subjects did not differ significantly in age, weight, or height. Dynamic posturography revealed statistically significantly different baseline presmoking measures in the patients with MS and the normal control subjects (Table I). In addition, statistically significant different responses to smoking a single marijuana cigarette were obtained in the patients with MS and the normal control subjects (Table II). It was observed in patients with MS at baseline that the severity of the myelopathy seemed to correlate with the degree of posturographic abnormality. Table I lists in order of significance those features of the parametric models in which the normal subjects differed significantly from the patients with MS. 
Table II. Significant marijuana treatment parameters from ANOVA analysis*

\begin{tabular}{|c|c|c|c|c|c|c|c|c|c|c|c|c|}
\hline & \multicolumn{6}{|c|}{ Patients } & \multicolumn{6}{|c|}{ Normal subjects } \\
\hline & \multicolumn{2}{|c|}{ Presmoking } & \multicolumn{2}{|c|}{ Placebo } & \multicolumn{2}{|c|}{ Marijuana } & \multicolumn{2}{|c|}{ Presmoking } & \multicolumn{2}{|c|}{ Placebo } & \multicolumn{2}{|c|}{ Marijuana } \\
\hline & $\overline{E O}$ & $E C$ & $\overline{E O}$ & $\overline{E C}$ & $E O$ & $E C$ & $E O$ & $\overline{E C}$ & $E O$ & $\overline{E C}$ & $E O$ & $E C$ \\
\hline Noise var & 1.02 & 4.83 & 0.72 & 3.27 & $1.67 \dagger$ & $5.73 \dagger$ & 0.59 & 1.93 & 0.59 & 3.20 & $0.79 \dagger$ & $2.80 \dagger$ \\
\hline Total phase lag & 4.06 & 3.77 & 3.74 & 3.41 & 4.19 & $5.55 \dagger$ & 4.18 & 3.67 & 5.04 & 3.27 & 4.2 & 3.41 \\
\hline
\end{tabular}

EO, Eyes open; EC, eyes closed.

* Numbers in table are group means.

$\uparrow$ Treatment factor (marijuana smoking) significant at $p=0.0025$ for comparison of premarijuana versus postmarijuana.

Transfer function and noise modalities. In normal subjects (Table I, columns 3 and 4), the damping effect of vision on postural responses was seen in the smaller $0.2 \mathrm{~Hz}$ gains and smaller variances (signal, noise, and residual) for the eyes-open responses. Vision had a reduced damping effect on the patient postural responses (Table I, columns 1 and 2). The signal variance, noise variance, and dead time were all much larger for patients than for normal subjects with both eyes open and eyes closed (Table I, Health).

Effects of marijuana smoking. All of the subjects (patients with MS and normal control subjects) obtained a more obvious psychologic "high" from the active than extracted marijuana cigarette. In both the patients with MS and age-matched control subjects, there were no gross changes in the neurologic examination after active marijuana smoke inhalation or extracted marijuana smoke inhalation. Two features of the parametric models were strongly affected by the presence of active marijuana smoke (Table II). In both the eyes-open and eyes-closed paradigms, for both normal subjects and patients, noise variance values, when compared with their respective presmoking control states, increased with active marijuana smoke inhalation. This effect was greatest for the patients. For the eyes-closed paradigm, smoking active marijuana increased the phase lags with which patients tracked the platform. A corresponding increase in phase lag from baseline was not observed for the normal subjects.

\section{DISCUSSION}

Dynamic posturography is a technique that has been shown to be sensitive to alterations in postural control mechanisms in patients with MS. Parker and Lerich ${ }^{10}$ reported dynamic posturography monitoring of balance before and during intravenous corticotropin and methylprednisolone treatment. The authors thought that dynamic posturography was a quantifiable objective technique that documented the worsening and im- provement in neurologic functioning that occurred with treatment. ${ }^{10}$

Surprisingly, neither extracted marijuana nor active marijuana smoke inhalation had a discernible effect in patients or control subjects on standard clinical neurologic examination of motor, sensory, cerebellar, or reflex testing. Patients often had the subjective feeling that they were clinically improved, yet postural responses of both normal subjects and patients were adversely affected in terms of noise variance; the negative effect was greatest in the patients. This observation provides evidence that single exposures to marijuana smoke interfere sufficiently with sensorymotor signal processing to cause even the normal subjects with visual stabilization to revert to a safer postural strategy than the energy minimizing one. Smoking marijuana increased the response delay in patients with eyes closed but not in the normal subjects. This may be caused by interference with sensory processing when visual cues are removed.

Petro and Ellenberger ${ }^{11}$ reported on the effects of $\Delta^{9}$-THC on spasticity in nine patients. Four patients responded to $\Delta^{9}$-THC with significant improvement of a spasticity score composed of reflexes, tone, and Babinski signs. In four patients the visual electromyographic interference pattern was reduced after $\Delta^{9}$. THC. Three patients reported feeling better, but only two had objective improvement. Clifford ${ }^{12}$ reported two severely disabled patients with MS with ataxia and tremor whose tremor but not ataxia was objectively improved. Five of six other patients had subjective, but no objective, improvement.

Smoking marijuana is a rapid and convenient method of administering $\Delta^{9}$-THC, its active ingredient. However, smoking suffers from variability in inhaling and, hence, variability in dosage. Dose-effect relationships are obscured. However, it is pertinent to determine if smoking just one marijuana cigarette in amounts sufficient to produce a state of mild intoxication has any effect on postural control. 
Dynamic posturography is a precise, quantitative functional assessment of the body's response to perturbational movements that are encountered as a normal part of living. It is not a measure of spasticity. Dynamic posturography requires integration of individual components of the motor, sensory, vestibular, visual cerebral, and cerebellar systems. It is a more functional assessment of the body's response to pertubational movements that are encountered as a normal part of life than isolated gait, motor, sensory, reflex, or coordination testing. Marijuana smoking worsened the dynamic posturography of both the patients and the control subjects. Therefore the hypothesis that initiated this research was not confirmed. The subclinical posturographic changes observed in ambulatory patients with MS under the influence of marijuana lessen their ability to react to their environment. Obviously, marijuana should not be used in a spastic ambulant population because the spasticity reducing effect of marijuana or its active ingredient $\Delta^{9}$-THC reported by others is not translated into an improvement in responding to changes in postural control.

An intriguing question to be answered in future research is whether chronic daily marijuana smoking for weeks would lead to differential tolerance development, with tolerance to the body balance deficit while an improvement in spasticity was maintained.

\section{References}

1. Consroe P, Snider SR. Therapeutic potential of cannabinoids in neurological disorders. In: Mechoulam R, ed. Cannabinoids as therapeutic agents. Boca Raton, Florida: CRC Press, 1986:21.

2. Consroe P, Sandyk R. Chapter 12. Potential role of cannabinoids for therapy of neurological disorders. In: Murphy L, Barke A, eds. Marijuana/cannabinoids, neurobiology and neurophysiology. Boca Raton, Florida: CRC Press, 1992:459-524.

3. Kiplinger GF, Manno JE, Rodda BE, Forney RB. Dose-response analysis of the effects of tetrahydrocannabinol in man. Clin Pharmacol Ther 1971;12:650-7.

4. Andres RO, Anderson DJ. Designing a better postural measurement system. Am J Otol 1980;1:197-206.

5. Pugh JE, Greenberg HS, Anderson DJ, Andres RO, Werness SAS. A computerized dynamic platform system for assessment of posture in neurologic patients [Abstract]. Neurology 1981;31(suppl 2):95.

6. Anderson DJ, Reschke MF, Homick JE, Werness SAS. Dynamic posture analysis of Spacelab-1 crew members. Exp Brain Res 1986;64:380-91.

7. Andres RO. A postural measurement system for induced body sway assessment [PhD Thesis]. Ann Arbor: University of Michigan, 1979.

8. Werness SAS, Anderson DJ. A computer program for linear non-parametric and parametric identification of biological data. Comput Programs Biomed 1984;18:7793.

9. Jaffe JH. Drug Addiction and Drug Abuse. In: Gilman AG, Goodman LS, Gilman A, eds. The pharmacologic basis of therapeutics. 6th ed. New York: MacMillan, 1980;535-84.

10. Parker SW, Lerich JR. Quantitative serial measurement of standing balance by dynamic posturography to monitor treatment of multiple sclerosis patients [Abstract]. Neurology 1990;40(suppl 1): 140.

11. Petro DJ, Ellenberger $C$. Treatment of human spasticity with $\Delta^{9}$-tetrahydrocannabinol. J Clin Pharmacol 1981; 21:4135-65.

12. Clifford DB. Tetrahydrocannabinol for treatment of multiple sclerosis. Am Neurol 1983;13:669-71. 